2. M. Ikeda and T. Nakayama, Supplementary remarks on Frobeniusean algebras, II, Osaka Math. J. vol. 2 (1950) pp. 7-12.

3. T. Nakayama, On Frobeniusean algebras, II, Ann. of Math. vol. 42 (1941) pp. $1-21$.

OSAKa UNIVERSITY AND

NAGOYA UNIVERSITY

\title{
THE NUMBER OF SUBGROUPS OF GIVEN INDEX IN NONDENUMERABLE ABELIAN GROUPS
}

\section{W. R. SCOTT}

Let $G$ be an Abelian group of order $A>\boldsymbol{\aleph}_{0}$. It has been shown [4, Theorem 9] that there exist $2^{A}$ subgroups of $G$ of order $A$, and that the intersection of all such subgroups is 0 . In this paper, this result is improved to the following: If $\boldsymbol{\aleph}_{0} \leqq B \leqq A$ and $A>\boldsymbol{\aleph}_{0}$, then an Abelian group of order $A$ has $2^{A}$ subgroups of index $B$, and the intersection of all such subgroups is 0 . In addition, it is shown that there is a set of $2^{A}$ subgroups $H_{\alpha}$ of index $B$ such that $G / H_{\alpha} \cong G / H_{\alpha^{\prime}}$ for all $\alpha, \alpha^{\prime}$.

Baer [1, p. 124] showed that if $G$ is an Abelian p-group which is the direct sum of $A$ cyclic groups of bounded order, then $G$ has $2^{A}$ subgroups of index $p$ (here $A$ may equal $\aleph_{0}$ ). The proof in the present paper is accomplished by extending Baer's result in an obvious manner to a wider class of $p$-groups, and then reducing all other cases to this one.

We shall use + and $\sum$ to denote direct sums, and $o(S)$ to denote the number of elements in $S$.

Lemma. Let $H \neq 0$ be an Abelian group, and let $G=\sum H_{\alpha}, \alpha \in S$, $H_{\alpha} \cong H$ for all $\alpha \in S, o(S)=A \geqq \boldsymbol{N}_{0}$. Then there are at least $2^{A}$ subgroups $K_{\beta}$ of $G$ such that $G / K_{\beta} \cong H$.

Proof. (This proof is the same as Baer's, and is included only for the sake of completeness.) Identify $H_{\alpha}$ with $H$. Let $\epsilon_{\alpha}$ be 0 or 1 for each $\alpha, \alpha \in S$. Let $K$ be the set of elements of $G$ such that $h_{\alpha_{0}}=\sum \epsilon_{\alpha} h_{\alpha}$, $\alpha \neq \alpha_{0}$. Then it is easy to verify that $K$ is a subgroup of $G$ and $G=H_{\alpha_{0}}$ $+K$. If $\epsilon_{\alpha}=0$, then $H_{\alpha} \subset K$, but if $\epsilon_{\alpha}=1$, then $H_{\alpha} \cap K=0$. Thus all of the $K^{\prime}$ 's are distinct, and the lemma is proved.

Presented to the Society, September 3, 1953; received by the editors March 2, 1953. 
Theorem. Let $G$ be an Abelian group of order $A>\boldsymbol{\aleph}_{0}$, and let $\boldsymbol{\aleph}_{0} \leqq B$ $\leqq$. Then

(i) there are exactly $2^{A}$ subgroups $H_{\alpha}$ of index $B$ and order $A$,

(ii) the intersection of the subgroups in (i) is 0 ,

(iii) there exist $2^{A}$ subgroups $K_{\beta}$, of index $B$ and order $A$, such that $G / K_{\beta} \cong G / K_{\beta^{\prime}} \cong \sum C_{\gamma}$ where either

(a) all $C_{\gamma}$ are cyclic of prime order, or

(b) all $C_{\gamma}$ are $p^{\infty}$ groups ( $p$ not necessarily fixed).

Remarks. The condition that $o\left(H_{\alpha}\right)=A$ or $o\left(K_{\beta}\right)=A$ is automatically satisfied by subgroups of index $B$ unless $B=A$. If (ii) is true, then it is clear that there is a set of $A$ subgroups, each of index $B$ and order $A$, whose intersection is 0 . Finally, since there are at most $2^{A}$ subgroups of $G$, (iii) implies (i), hence only (ii) and (iii) need be proved.

Proof. Case 1. $G=\sum C_{\gamma}, \gamma \in S, o\left(C_{\gamma}\right)=p, p$ a fixed prime. Then $o(S)=A$. By omitting $B$ and retaining $A$ summands, one obtains a subgroup $K$ of index $B$ and order $A$. By the lemma, $K$ has $2^{A}$ subgroups $K_{\beta}$ of index $p$ in $K$. The $K_{\beta}$ are thus of order $A$ and of index $B$ in $G$, and (iii) is satisfied. Since any given summand could have been omitted in obtaining $K$, (ii) is satisfied.

Case 2. $G=\sum C_{\gamma}, \gamma \in S$, where $C_{\gamma}$ is cyclic of order $p^{n \gamma}$. Again $o(S)=A$, and $G / p G$ is of the type considered in Case 1 . Hence there are $2^{A}$ subgroups $K_{\beta}^{*}$ of $G / p G$ as in (iii). Therefore there are $2^{A}$ subgroups $K_{\beta}$ of $G$ satisfying (iii). To obtain (ii), first omit from $G$ one summand containing a nonzero component of a given nonzero element $g$. Since the group $G^{*}$ thus obtained has finite index in $G$, one may then proceed as above.

Case 3. $G=\sum C_{\gamma}, \gamma \in S$, where $C_{\gamma}$ is a $p^{\infty}$ group, $p$ fixed. The proof is nearly identical to that in Case 1 .

Case $4 . G$ is a $p$-group. Then [3, Theorem 6] there exists a pure (= servant) subgroup $M$ of $G$ such that $(\alpha) M=\sum C_{\gamma}, \gamma \in S$, where $C_{\gamma}$ is cyclic, and $(\beta) G / M=\sum D_{\delta}$, where the $D_{\delta}$ are $p^{\infty}$ groups. If $\gamma_{1}, \cdots, \gamma_{n} \in S$, then $C_{\gamma_{1}}+\cdots+C_{\gamma_{n}}$ is a pure subgroup of $M$ since it is a direct summand thereof, consequently $C_{\gamma_{1}}+\cdots+C_{\gamma_{n}}$ is a pure subgroup of $G$. But since it is also of bounded order, we have [2, Theorem 5] $(\gamma) C_{\gamma_{1}}+\cdots+C_{\gamma_{n}}$ is a direct summand of $G$ for all $\gamma_{1}, \cdots, \gamma_{n} \in S$.

If $o(G / M)=A$, then by $(\beta)$ and Case 3, (iii) is satisfied for $G / M$, hence also for $G$.

If $o(G / M)<A$, then $o(M)=A$, and if $V$ is a set of representatives of the cosets of $M$, then the subgroup $L$ generated by $V$ has order less than $A$, and $M \cup L=G$. The summands $C_{\gamma}$ containing any com- 
ponent of any element of $M \cap L$ are fewer than $A$ in number. Hence there exists a subgroup $N$ of $M$ such that $N \supset M \cap L, o(M / N)=A$, and $M / N$ is isomorphic to a direct sum of some of the $C_{\gamma}$. Therefore by Case 2, (iii) is true for $M / N$, and therefore there are $2^{A}$ subgroups $K_{\beta}^{*}$ of $M$ containing $N$, and therefore $M \cap L$, such that the factor groups $M / K_{\beta}^{*}$ are as in (iii). It then follows from the isomorphism theorem that (iii) is satisfied for $G$.

To prove (ii), note that by $(\gamma)$, any element of $M$ may be omitted by a subgroup of finite index in $G$, hence from the above, by a subgroup $H_{\alpha}$ of index $B$ and order $A$. If $o(G / M)>B$, then by $(\beta)$ and Case 3 (perhaps for a cardinal smaller than $A$ ), any nonzero element of $G / M$ may be omitted by a subgroup of order $o(G / M)$ and of index $B$ in $G / M$, hence any element of $G$ outside of $M$ may be omitted by a subgroup of order $o(G / M) o(M)=A$ and of index $B$ in $G$. The same is true if $o(G / M)=B=A$. If $o(G / M)<B$ or $o(G / M)=B<A$, then any subgroup of $M$ of order $A$ and index $B$ in $M$ (omit $B$ and keep $A$ summands $C_{\gamma}$ in $(\alpha)$ ) has the property of omitting all elements outside $M$ and of having the right order and index in $G$.

Case 5. $G$ is periodic. Then $G=\sum G_{p}$, where $G_{p}$ is the $p$-component of $G$, and $A=\sum o\left(G_{p}\right)$. Let

$$
\begin{aligned}
& S_{1}=\left\{p \mid o\left(G_{p}\right) \leqq \aleph_{0}\right\}, \\
& S_{2}=\left\{p \mid o\left(G_{p}\right)>\aleph_{0}, \text { and (iii)(a) holds for } G_{p}\right\}, \\
& S_{3}=\left\{p \mid o\left(G_{p}\right)>\aleph_{0}, \text { and (iii)(a) does not hold for } G_{p}\right\}, \\
& N_{i}=\sum_{p \in S_{i}} G_{p}, \quad i=1,2,3 .
\end{aligned}
$$

Then either $o\left(N_{2}\right)=A$ or $o\left(N_{3}\right)=A$. Now if $p \in S_{2}$, then by Case 4, $G_{p}$ has $2^{o\left(G_{p}\right)}$ subgroups $K_{p, \gamma}$ of order $o\left(G_{p}\right)$ and index $\min \left(o\left(G_{p}\right), B\right)$ such that the $G_{p} / K_{p, \gamma}$ are as in (iii)(a). Hence if $K=N_{1}+N_{3}$ $+\sum K_{p, \gamma}, p \in S_{2}$, then $G / K$ is isomorphic to a fixed product of type (a) for all choices of $K$, and $K$ is of order $A$ and of index $\min \left(o\left(N_{2}\right), B\right)$ in $G$. There are

$$
\prod_{p \in S_{2}} 2^{\circ\left(G_{p}\right)}=2^{\Sigma_{p} \in S_{1} \circ\left(G_{p}\right)}=2^{\circ\left(N_{2}\right)}
$$

such $K$. Similarly there are (at least) $2^{o\left(N_{3}\right)} K_{\beta}$ such that $K_{\beta}$ is of order $A$, of index min $\left(o\left(N_{3}\right), B\right)$ in $G$, and such that $G / K_{\beta}$ is of type (b). Hence (iii) is satisfied, since either $o\left(N_{2}\right)$ or $o\left(N_{3}\right)$ equals $A$.

To prove (ii), let $g \in G$ have component $g_{p_{0}} \neq 0$. If $o\left(G_{p_{0}}\right)<B$, or $o\left(G_{p_{0}}\right)=B<A$, then omit the summand $G_{p_{0}}$ from $G$, and find a subgroup of order $A$ and of index $B$ in the resulting $G^{*}$. If $o\left(G_{p_{0}}\right)>B$ or $o\left(G_{p_{0}}\right)=B=A$, then by Case 4 , there exists a subgroup $K_{p_{0}}$ of $G_{p_{0}}$ 
of index $B$ in $G_{p_{0}}$ and, in case $B=A$, of order $A$, which omits $g_{p_{0}}$. Then $K_{p_{0}}+\sum G_{p}, p \neq p_{0}$, omits $g$ and has the required properties. Thus (ii) holds.

Case 6. $G$ is not periodic. Let $F=\left\{f_{\alpha}\right\}$ be a maximal independent set of elements of $G$. Let $L_{k}, k=2,3, \cdots$, be the subgroup generated by the maximal independent set of elements $\left\{k f_{\alpha}\right\}$. If $o(F)<A$, then $o\left(L_{k}\right)<A$ and $o\left(G / L_{k}\right)=A$. If $o(F)=A$, then the $f_{\alpha}$ lie in distinct cosets of $L_{k}$, so again $o\left(G / L_{k}\right)=A$. But $G / L_{k}$ is periodic, and therefore by Case 5, (iii) is true, and in (ii), $\cap H_{\alpha} \subseteq L_{k}$. Since $\cap L_{k}=0$. $k=2,3, \cdots$, (ii) also holds.

\section{BIBLIOGRAPHY}

1. R. Baer, Dualism in abelian groups, Bull. Amer. Math. Soc. vol. 43 (1937) pp. 121-124.

2. L. Koulikoff, Zur Theorie der abelschen Gruppen von beliebiger Mächtigkeit, Mat. Sbornik vol. 9 (1941) pp. 165-181 (Russian with Germany summary).

3. - On the theory of abelian groups of arbitrary power, Mat. Sbornik vol. 16 (1945) pp. 129-162 (Russian with English summary).

4. W. R. Scott, Groups and cardinal numbers, Amer. J. Math. vol. 74 (1952) pp. 187-197.

UNIVERSITY OF KANSAS 